BI-TP 2007/14

MS-TP-07-16

arXiv:0707.2458

\title{
Thermal imaginary part of a real-time static potential from classical lattice gauge theory simulations
}

\author{
M. Laine ${ }^{\mathrm{a}}$, O. Philipsen ${ }^{\mathrm{b}}$, M. Tassler ${ }^{\mathrm{b}}$ \\ ${ }^{a}$ Faculty of Physics, University of Bielefeld, D-33501 Bielefeld, Germany \\ ${ }^{\mathrm{b}}$ Institute for Theoretical Physics, University of Münster, D-48149 Münster, Germany
}

\begin{abstract}
Recently, a finite-temperature real-time static potential has been introduced via a Schrödinger-type equation satisfied by a certain heavy quarkonium Green's function. Furthermore, it has been pointed out that it possesses an imaginary part, which induces a finite width for the tip of the quarkonium peak in the thermal dilepton production rate. The imaginary part originates from Landau-damping of low-frequency gauge fields, which are essentially classical due to their high occupation number. Here we show how the imaginary part can be measured with classical lattice gauge theory simulations, accounting non-perturbatively for the infrared sector of finite-temperature field theory. We demonstrate that a non-vanishing imaginary part indeed exists non-perturbatively; and that its value agrees semi-quantitatively with that predicted by Hard Loop resummed perturbation theory.
\end{abstract}

September 2007 


\section{Introduction}

The notion of a static potential, generalizing the potential that appears in the Schrödingerequation of non-relativistic quantum mechanics, is thought to play a role for heavy quarkonium physics in QCD. If the energy of a two-quark system, $E$, is close to twice the mass of the heavy quark, $M$, so that the combined "kinetic energy" of the two quarks, $E-2 M$, is small compared with $M$, then we may assume the quarks to be "static" to a good approximation, moving only slowly in the attractive potential generated by the colour fields.

To turn this intuitive picture into a quantitative description requires the use of effective field theory methods. At zero temperature various energy and momentum scales can be identified, the small expansion parameter being related to the ratio $(E-2 M) / M$. The relevant effective theory is called NRQCD [1], or one of its variants, like pNRQCD [2]; for reviews on the various effective theories used for describing heavy quarkonium, see refs. [3, 4]. The static potential plays the role of a certain matching coefficient in these effective theories: it is related to, but not identical with, the non-perturbative static potential that is traditionally defined from a large Euclidean Wilson loop in lattice QCD.

At finite temperatures, the situation becomes more complicated than at zero temperature. Indeed, finite-temperature field theory possesses many momentum and energy scales of its own: gluonic momenta could parametrically be $k \sim \pi T, g T, g^{2} T$ [5, 6], while gluonic frequencies (energies) can be even softer, down to $E \sim g^{4} T$ [7, 8]. Here $T$ is the temperature and $g$ is the QCD gauge coupling. The relevant effective description now depends on the relation of these scales to the scales already appearing in the zero-temperature situation.

In fact, at finite temperatures, the situation is quite complicated even at the leading nontrivial order in $g$. This might be anticipated from the fact alone that the definition of a static potential based on the Euclidean Wilson loop appears to lose its meaning: the Euclidean time direction becomes compact, and large Wilson loops do not possess the same interpretation as at zero temperature. Replacing the Wilson loop by a correlator of Polyakov loops does not remedy the situation [9, 10]. Moreover, physics lives in Minkowski spacetime, which at finite temperatures in general requires a non-trivial analytic continuation [11.

Recently an attempt was made to give a proper definition of a static potential in this situation, in the sense of obtaining an object which has a direct connection to the spectral function of the heavy quarkonium system (at least up to some order in perturbation theory) [12]. Formally, the static potential could be defined as a certain coefficient in the large- $M$ expansion of an equation of motion satisfied by a suitable heavy quarkonium Green's function. At leading non-trivial order, the corresponding object was computed in Hard Thermal Loop [13. resummed perturbation theory in ref. [12]. It was found that, at least to this order, the static potential can also be obtained from a specific analytic continuation of the Wilson loop defined in Euclidean spacetime with a compact time direction. At the same time, this analytic continuation yields properties that are not familiar from the zero-temperature context: in particular, the potential develops an imaginary part. 
It is the purpose of the present paper to elaborate on the existence of an imaginary part. We start, in Sec. 2, by reviewing the definition(s) introduced in ref. [12. In Sec. 3 we argue that the imaginary part of the static potential remains non-zero in the classical limit, by computing it perturbatively in classical lattice gauge theory. Given that perturbative computations at finite temperatures may ultimately suffer from infrared divergences, we carry out non-perturbative Monte Carlo simulations in classical lattice gauge theory in Sec. 4, and compare the results with those of the perturbative computation. We conclude in Sec. 5,

\section{Definition of a real-time static potential}

We start by defining a certain Green's function in hot QCD. Let $\mathbf{r}$ be a point-splitting vector, and $\hat{\psi}$ a generic heavy quark field operator in the Heisenberg picture. Then we introduce

$$
\check{C}_{>}(t, \mathbf{r}) \equiv \int \mathrm{d}^{3} \mathbf{x}\left\langle\hat{\bar{\psi}}\left(t, \mathbf{x}+\frac{\mathbf{r}}{2}\right) \gamma^{\mu} W \hat{\psi}\left(t, \mathbf{x}-\frac{\mathbf{r}}{2}\right) \hat{\bar{\psi}}(0, \mathbf{0}) \gamma_{\mu} \hat{\psi}(0, \mathbf{0})\right\rangle,
$$

where $W$ is a Wilson line along a straight path connecting the adjacent operators, inserted in order to keep the Green's function gauge-invariant; the metric is $\eta_{\mu \nu}=\operatorname{diag}(+---)$; and the expectation value refers to $\langle\ldots\rangle \equiv \mathcal{Z}^{-1} \operatorname{Tr}[\exp (-\hat{H} / T)(\ldots)]$, where $\mathcal{Z}$ is the partition function, $\hat{H}$ is the QCD Hamiltonian operator, and $T$ is the temperature.

The significance of the Green's function in Eq. (2.1) is that if we take the limit $\mathbf{r} \rightarrow \mathbf{0}$, and subsequently Fourier transform with respect to the time $t$, then we obtain a function which is trivially related to the heavy quarkonium spectral function, $\rho(\omega)$, in the vector channel:

$$
\rho(\omega)=\frac{1}{2}\left(1-e^{-\frac{\omega}{T}}\right) \int_{-\infty}^{\infty} \mathrm{d} t e^{i \omega t} \check{C}_{>}(t, \mathbf{0}) .
$$

On the other hand, keeping $\mathbf{r} \neq \mathbf{0}$ for the moment, makes it easier to analyse this Green's function in perturbation theory.

Let us consider $\check{C}_{>}(t, \mathbf{r})$ in the limit that the heavy quark mass $M$ is very large. Then $\check{C}_{>}(t, \mathbf{r})$ satisfies a Schrödinger equation of the type

$$
\left\{i \partial_{t}-\left[2 M+V_{>}(t, r)-\frac{\nabla_{\mathbf{r}}^{2}}{M}+\mathcal{O}\left(\frac{1}{M^{2}}\right)\right]\right\} \check{C}_{>}(t, \mathbf{r})=0,
$$

with the initial condition

$$
\check{C}_{>}(0, \mathbf{r})=-6 N_{\mathrm{c}} \delta^{(3)}(\mathbf{r})+\mathcal{O}\left(\frac{1}{M}\right) .
$$

The terms shown explicitly in Eqs. (2.3), (2.4) result from a tree-level computation; they also develop multiplicative radiative corrections which we have omitted for simplicity. In contrast, the potential denoted by $V_{>}(t, r)$ originates only at 1-loop order. It can be defined as the coefficient scaling as $\mathcal{O}\left(M^{0}\right)$, after acting on $\check{C}_{>}(t, \mathbf{r})$ with the time derivative $i \partial_{t}$. 
Now, as Eq. (2.3) shows, $V_{>}(t, r)$ can even be defined in the limit $M \rightarrow \infty$, provided that the trivial factor $2 M$ is shifted away by a redefinition of time, as is standard in NRQCD. In ref. [12], the computation in this limit was carried out to 1-loop order, $\mathcal{O}\left(g^{2}\right)$, in Hard Thermal Loop resummed perturbation theory [13. It was found that at this order $V_{>}(t, r)$ can in fact be extracted from the equation

$$
i \partial_{t} C_{E}(i t, r) \equiv V_{>}(t, r) C_{E}(i t, r),
$$

where the function $C_{E}(\tau, r)$ is nothing but the Euclidean Wilson loop, computed with an imaginary time coordinate $\tau$, with gauge fields periodic in $\tau \rightarrow \tau+\hbar / T$.

The expression that was obtained for $V_{>}(t, r)$ in ref. [12] reads (the superscript refers to the order in $g$; we keep $\hbar \neq 1$; and we assume the use of dimensional regularization)

$$
\begin{aligned}
V_{>}^{(2)}(t, r) & =-\frac{g^{2} C_{F} \hbar}{4 \pi}\left[m_{\mathrm{D}}+\frac{\exp \left(-m_{\mathrm{D}} r\right)}{r}\right]+\delta V_{>}^{(2)}(t, r), \\
\delta V_{>}^{(2)}(t, r) & =g^{2} C_{F} \hbar \int \frac{\mathrm{d}^{3} \mathbf{p}}{(2 \pi)^{3}}\left(1-\cos p_{3} r\right) \times \\
& \times \int_{-\infty}^{\infty} \frac{\mathrm{d} p^{0}}{\pi} p^{0}\left[e^{-i\left|p^{0}\right| t}+n_{\mathrm{B}}\left(\left|p^{0}\right|\right)\left(e^{-i\left|p^{0}\right| t}-e^{i\left|p^{0}\right| t}\right)\right] \times \\
& \times\left[\left(\frac{1}{\mathbf{p}^{2}}-\frac{1}{\left(p^{0}\right)^{2}}\right) \rho_{E}\left(p^{0}, \mathbf{p}\right)+\left(\frac{1}{p_{3}^{2}}-\frac{1}{\mathbf{p}^{2}}\right) \rho_{T}\left(p^{0}, \mathbf{p}\right)\right] .
\end{aligned}
$$

Here $C_{F} \equiv\left(N_{\mathrm{c}}^{2}-1\right) / 2 N_{\mathrm{c}} ; m_{\mathrm{D}}$ is the Debye mass parameter (actually of dimensionality $1 /$ distance rather than mass); $n_{\mathrm{B}}(x) \equiv 1 /[\exp (\hbar x / T)-1]$ is the Bose distribution function; and we have chosen $\mathbf{r} \equiv(0,0, r)$. The $r$-independent term in Eq. (2.6) amounts to twice a thermal mass correction for the heavy quark. For the gluon spectral functions $\rho_{E}, \rho_{T}$ we assume the conventions specified in appendix A of ref. [14].

It can be observed that Eqs. (2.6), (2.7) contain both a real and an imaginary part. In particular, the familiar-looking structure in Eq. (2.6), representing a Debye-screened Coulomb potential, is real, while the manifestly "thermal" part in Eq. (2.7), containing $n_{\mathrm{B}}$, is purely imaginary. As pointed out in ref. [12, this purely imaginary term remains non-zero in the limit $t \rightarrow \infty$, because of Bose-enhancement at small frequencies, $\hbar\left|p^{0}\right| \ll T$ (cf. Eq. (2.9)).

The Bose-enhanced term corresponds to the physics of the classical limit $\hbar \rightarrow 0$, in which situation $n_{\mathrm{B}}\left(\left|p^{0}\right|\right)=T / \hbar\left|p^{0}\right|$. In fact, all other terms vanish in this limit, being multiplied by $\hbar$. The classical potential then reads

$$
\begin{aligned}
V_{\mathrm{cl}}^{(2)}(t, r) & =g^{2} C_{F} T \int \frac{\mathrm{d}^{3} \mathbf{p}}{(2 \pi)^{3}}\left(1-\cos p_{3} r\right) \int_{-\infty}^{\infty} \frac{\mathrm{d} p^{0}}{\pi}\left(e^{-i p^{0} t}-e^{i p^{0} t}\right) \times \\
& \times \lim _{\hbar \rightarrow 0}\left[\left(\frac{1}{\mathbf{p}^{2}}-\frac{1}{\left(p^{0}\right)^{2}}\right) \rho_{E}\left(p^{0}, \mathbf{p}\right)+\left(\frac{1}{p_{3}^{2}}-\frac{1}{\mathbf{p}^{2}}\right) \rho_{T}\left(p^{0}, \mathbf{p}\right)\right],
\end{aligned}
$$

where we have also simplified the way in which $p^{0}$ 's appear. For large times,

$$
\lim _{t \rightarrow \infty} \frac{e^{i p^{0} t}-e^{-i p^{0} t}}{p^{0}}=2 \pi i \delta\left(p^{0}\right),
$$


and we obtain

$$
V_{\mathrm{cl}}^{(2)}(\infty, r)=2 i g^{2} C_{F} T \int \frac{\mathrm{d}^{3} \mathbf{p}}{(2 \pi)^{3}}\left(1-\cos p_{3} r\right) \lim _{p^{0} \rightarrow 0} \lim _{\hbar \rightarrow 0} \frac{\rho_{E}\left(p^{0}, \mathbf{p}\right)}{p^{0}} .
$$

If we also take the limit $r \rightarrow \infty$, the cosine-term in Eq. (2.10) drops out. Assuming for a moment that the two limits and the integration in Eq. (2.10) commute, and making use of the known Hard Thermal Loop form of $\rho_{E}\left(p^{0}, \mathbf{p}\right)$ (Eq. (B.13) of ref. [12] shows $\rho_{E}$ at small $\left|p^{0}\right|$ with our conventions), containing the parameter $m_{\mathrm{D}}^{2}$, then leads to the provisional result

$$
V_{\mathrm{cl}}^{(2)}(\infty, \infty) \stackrel{?}{=} \lim _{\hbar \rightarrow 0}-i g^{2} C_{F} T \int \frac{\mathrm{d}^{3} \mathbf{p}}{(2 \pi)^{3}} \frac{\pi m_{\mathrm{D}}^{2}}{|\mathbf{p}|\left(\mathbf{p}^{2}+m_{\mathrm{D}}^{2}\right)^{2}}=\lim _{\hbar \rightarrow 0}-i \frac{g^{2} C_{F} T}{4 \pi}=-i \frac{g^{2} C_{F} T}{4 \pi} .
$$

Note that in the quantum theory the same result is obtained for the asymptotic value $\delta V_{>}^{(2)}(\infty, \infty)$ [12], and in this sense Eq. (2.11) is indeed the correct physical expression.

Now, given that the integral in Eq. (2.11) is finite, it might be assumed that the result is independent of the regularization procedure. It turns out that this argument is too naive: in fact, $m_{\mathrm{D}}^{2}$ diverges as $g^{2} T^{2} / \hbar$ in dimensional regularization, indicating that the classical limit may introduce ultraviolet singularities. In particular, if the ultraviolet is regularized by a lattice rather than dimensionally, with a spatial lattice spacing $a$, then the limit becomes finite, $\lim _{\hbar \rightarrow 0} m_{\mathrm{D}}^{2} \propto g^{2} T / a$ [15, 16]. Thus the classical limit in Eq. (2.10) does exist, but the price to pay is that $\rho_{E}$ and subsequently $V_{\mathrm{cl}}^{(2)}(\infty, \infty)$ depend on the details of the regularization procedure. In particular, carrying out the limits in the order indicated by Eq. (2.10) with lattice regularization, does not lead to the expression in Eq. (2.11) (cf. Fig. 3 below).

Fortunately, this problem is not too serious: an analogous situation was met in studies of the sphaleron rate in the electroweak theory, yet classical lattice gauge theory simulations [17] did yield non-perturbative physical information, once properly interpreted (see, e.g., refs. [18, 19]). In our case, Fig. 3 implies that we cannot use classical lattice gauge theory simulations to compute corrections directly to Eq. (2.11). However, we can compute the analogues of Eqs. (2.8)-(2.11) with Hard Thermal Loop perturbation theory adapted to the ultraviolet physics of the classical lattice [15, 16, and compare subsequently these results (the dashed curve in Fig. 3) with a non-perturbative determination. In this way we can probe the infrared sector of thermal field theory, which indeed is classical in nature.

\section{Perturbative real-time static potential in classical lattice gauge theory}

We assume that the theory is regularized by introducing a cubic spatial lattice, while the time coordinate is continuous. Gauge field configurations are generated with a Wilson-discretised Hamiltonian (cf. Eq. (4.1) below), and evolved with the classical equations of motion (cf. Eqs. (4.3), (4.4) below). The results depend on a single parameter,

$$
\beta \equiv \frac{2 C_{A}}{g^{2} T a}
$$




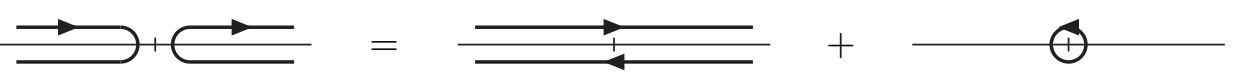

Figure 1: Integration contours for the classical real-time static potential.

where $C_{A} \equiv N_{\mathrm{c}}$.

The way to carry out perturbation theory in this situation was worked out in refs. [15, 16]. The procedure is analogous to Hard Thermal Loop resummed perturbation theory [13], with technical differences originating from the different ultraviolet physics. We will refer to this procedure as Hard Classical Loop (HCL) perturbation theory.

Let us start by introducing the notation

$$
\tilde{p}_{i} \equiv \frac{2}{a} \sin \left(\frac{a p_{i}}{2}\right), \quad \stackrel{\circ}{p}_{i} \equiv \frac{1}{a} \sin \left(a p_{i}\right), \quad \tilde{\mathbf{p}}^{2} \equiv \sum_{i=1}^{3} \tilde{p}_{i}^{2}, \quad \stackrel{\circ}{\mathbf{p}}^{2} \equiv \sum_{i=1}^{3} \stackrel{\circ}{p}_{i}^{2} .
$$

Also, the integration measure is denoted by

$$
\int \mathrm{d} \mathbf{p} \equiv \int_{-\pi / a}^{\pi / a} \frac{\mathrm{d}^{3} \mathbf{p}}{(2 \pi)^{3}}
$$

Then, we consider Eq. (2.8), with a few straightforward modifications following from the introduction of lattice regularization:

$$
\begin{aligned}
V_{\mathrm{cl}}^{(2)}(t, r) & =g^{2} C_{F} T \int \mathrm{d} \mathbf{p}\left(1-\cos p_{3} r\right) \int_{-\infty}^{\infty} \frac{\mathrm{d} p^{0}}{\pi}\left(e^{-i p^{0} t}-e^{i p^{0} t}\right) \times \\
& \times\left[\left(\frac{1}{\tilde{\mathbf{p}}^{2}}-\frac{1}{\left(p^{0}\right)^{2}}\right) \rho_{E}\left(p^{0}, \tilde{\mathbf{p}}\right)+\left(\frac{1}{\tilde{p}_{3}^{2}}-\frac{1}{\tilde{\mathbf{p}}^{2}}\right) \rho_{T}\left(p^{0}, \tilde{\mathbf{p}}\right)\right] .
\end{aligned}
$$

Here,

$$
\rho_{E}\left(p^{0}, \tilde{\mathbf{p}}\right) \equiv \frac{1}{2 i}\left[\Delta_{E}\left(p^{0}+i \epsilon, \tilde{\mathbf{p}}\right)-\Delta_{E}\left(p^{0}-i \epsilon, \tilde{\mathbf{p}}\right)\right],
$$

with $\epsilon=0^{+}$, and the propagator $\Delta_{E}$ has the form

$$
\Delta_{E}\left(p^{0}, \tilde{\mathbf{p}}\right)=\frac{1}{\tilde{\mathbf{p}}^{2}-\left(p^{0}\right)^{2}+\Pi_{E}\left(p^{0}, \tilde{\mathbf{p}}\right)} .
$$

The $\operatorname{limit}_{\hbar \rightarrow 0}$ is assumed everywhere but not shown explicitly.

\subsection{Behaviour at finite times}

In order to simplify Eq. (3.4), it is convenient, following ref. [20], to view the $p^{0}$-integration as an integral in the complex plane, and to deform the contour suitably. As it stands, the integrand in Eq. (3.4) is finite at $p^{0}=0$ (note that $\rho_{E}$ is linear in $p^{0}$ around the origin). 
However, the part multiplying $\rho_{E}$ contains a pole at $p^{0}=0$; this pole just does not contribute because of the mentioned property of $\rho_{E}$. Writing $\rho_{E}$ as in Eq. (3.5), this means that we can view the original integral as indicated in the left-most drawing in Fig. 1, and then also deform it accordingly. Subsequently, $\epsilon$ can be taken to be finite, because there are no singularities outside of the real axis. Furthermore, assuming $t>0$, terms multiplied by $\exp \left[i\left(p^{0}+i \epsilon\right) t\right]$ and $\exp \left[-i\left(p^{0}-i \epsilon\right) t\right]$ must vanish, because we can imagine taking $\epsilon$ arbitrarily large. The integrand can only decrease in this limit, and being multiplied by $\exp (-\epsilon t)$, the integral then vanishes (it is a good cross-check of the numerics to verify the vanishing at any finite $\epsilon$ ). Finally, the symmetry properties of the integrand allow to reflect the lower of the remaining contours to the upper half-plane. We thus obtain

$$
\begin{gathered}
\operatorname{Im}\left[\frac{V_{\mathrm{cl}}^{(2)}(t, r)}{g^{2} T}\right]=2 C_{F} \int \mathrm{d} \mathbf{p}\left(1-\cos p_{3} r\right)\left\{t \Delta_{E}(0, \tilde{\mathbf{p}})-\int_{-\infty}^{\infty} \frac{\mathrm{d} p^{0}}{2 \pi} e^{-i\left(p^{0}+i \epsilon\right) t} \times\right. \\
\left.\times\left[\left(\frac{1}{\tilde{\mathbf{p}}^{2}}-\frac{1}{\left(p^{0}+i \epsilon\right)^{2}}\right) \Delta_{E}\left(p^{0}+i \epsilon, \tilde{\mathbf{p}}\right)+\left(\frac{1}{\tilde{p}_{3}^{2}}-\frac{1}{\tilde{\mathbf{p}}^{2}}\right) \Delta_{T}\left(p^{0}+i \epsilon, \tilde{\mathbf{p}}\right)\right]\right\},
\end{gathered}
$$

where the first term is the contribution of the pole in Fig. 1.

Let us stress that the integration in Eq. (3.7) is independent of the value of $\epsilon>0$, since there are no poles in the upper half-plane. Checking the independence in practice offers another cross-check for the accuracy of the numerical integration. Naturally, small values of $\epsilon$ are difficult, because the integrand becomes strongly peaked around the origin, while large values of $\epsilon$ are also difficult, because the latter term is multiplied by $\exp (\epsilon t)$, whereby the numerical errors of the integration are exponentially amplified at large $t$; a useful compromise appears to be $\epsilon \simeq 1 / a$. In general, it is advantageous to decrease $\epsilon$ when increasing $t$.

In order to insert the propagators $\Delta_{E}, \Delta_{T}$, we need to know the self-energies $\Pi_{E}, \Pi_{T}$ (cf. Eq. (3.6) ). Starting from the spatial part of the gluon self-energy [15, 16]

$$
\Pi_{i j}\left(p^{0}, \tilde{\mathbf{p}}\right)=2 g^{2} T C_{A} \int \mathrm{d} \mathbf{q} \frac{1}{\tilde{\mathbf{q}}^{2}} \frac{p^{0} v_{i} v_{j}}{p^{0}-\tilde{\mathbf{p}} \cdot \mathbf{v}},
$$

with

$$
v_{i} \equiv \frac{\stackrel{\circ}{q}_{i}}{\sqrt{\tilde{\mathbf{q}}^{2}}},
$$

and employing the projection operators $P_{\mu \nu}^{E}, P_{\mu \nu}^{T}$ defining $\Pi_{E}, \Pi_{T}$ (we use the conventions specified in appendix B of ref. [12]), we obtain

$$
\begin{aligned}
& \Pi_{E}\left(p^{0}, \tilde{\mathbf{p}}\right)=2 g^{2} T C_{A}\left(1-\frac{\left(p^{0}\right)^{2}}{\tilde{\mathbf{p}}^{2}}\right)\left(\frac{\Sigma}{4 \pi a}-\int \mathrm{d} \mathbf{q} \frac{1}{\tilde{\mathbf{q}}^{2}} \frac{p^{0}}{p^{0}-\tilde{\mathbf{p}} \cdot \mathbf{v}}\right) \\
& \Pi_{T}\left(p^{0}, \tilde{\mathbf{p}}\right)=g^{2} T C_{A}\left[\frac{\left(p^{0}\right)^{2}}{\tilde{\mathbf{p}}^{2}}\left(\frac{\Sigma}{4 \pi a}-\int \mathrm{d} \mathbf{q} \frac{1}{\tilde{\mathbf{q}}^{2}} \frac{p^{0}}{p^{0}-\tilde{\mathbf{p}} \cdot \mathbf{v}}\right)+\int \mathrm{d} \mathbf{q} \frac{\stackrel{\mathbf{q}}{ }^{2}}{\left(\tilde{\mathbf{q}}^{2}\right)^{2}} \frac{p^{0}}{p^{0}-\tilde{\mathbf{p}} \cdot \mathbf{v}}\right] .
\end{aligned}
$$

Here

$$
\frac{\Sigma}{4 \pi a} \equiv \int \mathrm{d} \mathbf{q} \frac{1}{\tilde{\mathbf{q}}^{2}}
$$


where $\Sigma \approx 3.175911535625$ is a trigonometric factor which can be expressed in terms of the complete elliptic integral of the first kind [21]. Note that with the form in Eq. (3.10), the combination containing $\Delta_{E}$ in Eq. (3.7) becomes

$$
\left(\frac{1}{\tilde{\mathbf{p}}^{2}}-\frac{1}{\left(p^{0}+i \epsilon\right)^{2}}\right) \Delta_{E}\left(p^{0}+i \epsilon, \tilde{\mathbf{p}}\right)=-\frac{1}{\left(p^{0}+i \epsilon\right)^{2}} \frac{1}{\tilde{\mathbf{p}}^{2}+2 g^{2} T C_{A}\left(\frac{\Sigma}{4 \pi a}-\int \mathrm{d} \mathbf{q} \frac{1}{\frac{\tilde{\mathbf{q}}^{2}}{p^{0}-\tilde{\mathbf{p}} \cdot \mathbf{v}}}\right)}
$$

Denoting the square brackets in Eq. (3.7) by $\mathcal{I}\left(p^{0}+i \epsilon, \tilde{\mathbf{p}}\right)$, and making use of the properties

$$
\mathcal{I}\left(-p^{0}+i \epsilon, \tilde{\mathbf{p}}\right)=\mathcal{I}\left(p^{0}-i \epsilon, \tilde{\mathbf{p}}\right)=\left[\mathcal{I}\left(p^{0}+i \epsilon, \tilde{\mathbf{p}}\right)\right]^{*},
$$

the integral over $p^{0}$ can furthermore be reduced to regular cosine and sine transforms:

$$
\begin{aligned}
\int_{-\infty}^{\infty} \frac{\mathrm{d} p^{0}}{2 \pi} & e^{-i\left(p^{0}+i \epsilon\right) t} \mathcal{I}\left(p^{0}+i \epsilon, \tilde{\mathbf{p}}\right) \\
& =\frac{e^{\epsilon t}}{\pi} \int_{0}^{\infty} \mathrm{d} p^{0}\left\{\cos \left(p^{0} t\right) \operatorname{Re}\left[\mathcal{I}\left(p^{0}+i \epsilon, \tilde{\mathbf{p}}\right)\right]+\sin \left(p^{0} t\right) \operatorname{Im}\left[\mathcal{I}\left(p^{0}+i \epsilon, \tilde{\mathbf{p}}\right)\right]\right\} .
\end{aligned}
$$

Though efficient routines for such transforms exist, it is also clear that the accuracy requirements grow exponentially with $t$, so that very large times are difficult to reach.

In a practical lattice study, the system possesses not only a finite lattice spacing, but also a finite extent, $L=N a$, where $N$ is the number of lattice points. We assume that the box is cubic and that periodic boundary conditions are imposed in every direction. Furthermore, let us assume that we use changes of integration variables to write the momentum integrations over the "positive" octant only,

$$
\int_{-\pi / a}^{\pi / a} \frac{\mathrm{d} p_{i}}{2 \pi} \mathcal{F}\left(p_{i}\right)=\int_{0}^{\pi / a} \frac{\mathrm{d} p_{i}}{2 \pi} \mathcal{G}\left(p_{i}\right), \quad \mathcal{G}\left(p_{i}\right) \equiv\left[\mathcal{F}\left(p_{i}\right)+\mathcal{F}\left(-p_{i}\right)\right], \quad i=1,2,3 .
$$

In a finite volume this then goes over into

$$
\int_{0}^{\pi / a} \frac{\mathrm{d} p_{i}}{2 \pi} \mathcal{G}\left(p_{i}\right) \longrightarrow \frac{1}{N a}\left[\frac{1}{2} \mathcal{G}(0)+\sum_{i=1}^{N / 2-1} \mathcal{G}\left(\frac{2 \pi i}{N a}\right)+\frac{1}{2} \mathcal{G}\left(\frac{\pi}{a}\right)\right] .
$$

Note that in finite volume, the analytically known integral in Eq. (3.12) should also be replaced by a numerically evaluated sum.

Now, Eq. (3.17) contains also a contribution from the zero-mode, $p_{i}=0$. Its treatment requires in general some care. In $\Pi_{E}, \Pi_{T}$, loop momenta are by definition "hard": it is sensible (and in fact necessary) to leave out the zero-mode. In the remaining sum in Eq. (3.7), in contrast, momenta could be soft: we thus keep the contribution of the zero-mode as well (even though the practical effect is small). Note that for the zero-mode,

$$
\Pi_{E}\left(p^{0}, \mathbf{0}\right)=\Pi_{T}\left(p^{0}, \mathbf{0}\right)=\omega_{\mathrm{pl}}^{2}, \quad \omega_{\mathrm{pl}}^{2} \equiv \frac{2}{3} g^{2} T C_{A} \int \mathrm{d} \mathbf{q} \frac{\stackrel{\mathbf{q}}{ }^{2}}{\left(\tilde{\mathbf{q}}^{2}\right)^{2}},
$$




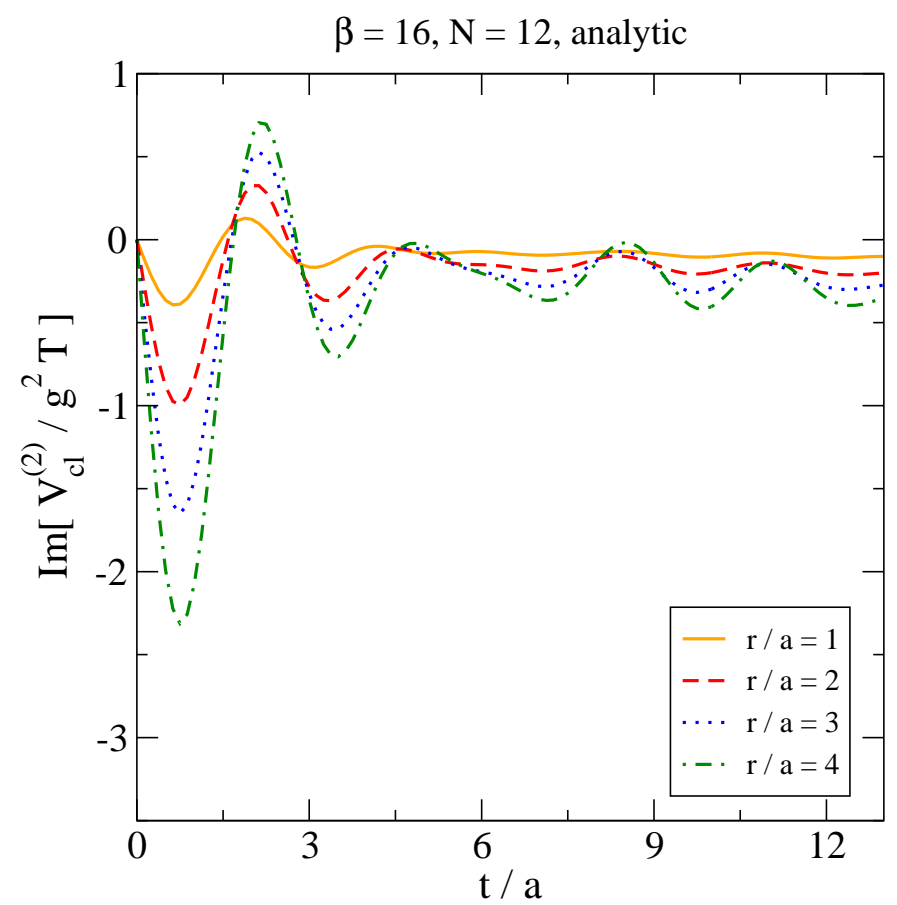

Figure 2: The imaginary part of the classical real-time static potential, to leading non-trivial order in HCL-resummed perturbation theory (Eq. (3.7) with $\int \mathrm{d} \mathbf{p}$ replaced by a finite-volume sum), for $\beta=16, N=12, N_{\mathrm{c}}=3$.

where the integration can be replaced by a sum (without zero-mode) as before 1 Then the combination in Eq. (3.7) becomes

$$
\begin{aligned}
& \left(1-\cos p_{3} r\right)\left[\left(\frac{1}{\tilde{\mathbf{p}}^{2}}-\frac{1}{\left(p^{0}+i \epsilon\right)^{2}}\right) \frac{1}{\tilde{\mathbf{p}}^{2}-\left(p^{0}+i \epsilon\right)^{2}+\omega_{\mathrm{pl}}^{2}}+\left(\frac{1}{\tilde{p}_{3}^{2}}-\frac{1}{\tilde{\mathbf{p}}^{2}}\right) \frac{1}{\tilde{\mathbf{p}}^{2}-\left(p^{0}+i \epsilon\right)^{2}+\omega_{\mathrm{pl}}^{2}}\right] \\
& \stackrel{\mathbf{p}=\mathbf{0}}{\longrightarrow} \frac{r^{2}}{2} \frac{1}{-\left(p^{0}+i \epsilon\right)^{2}+\omega_{\mathrm{pl}}^{2}} .
\end{aligned}
$$

In practice, for the values $N \geq 12$ that we have used, finite-volume effects are almost invisible at small times. Perturbative finite-volume effects grow rapidly with time, however, and also with distance. On the other hand, perturbation theory tends to overestimate their significance, since it lacks the mass gap generated by the confining dynamics. At the same time, whenever justified, it appears to be numerically advantageous to use the finite-volume expressions, which contain a six-fold exact sum, rather than to approximate the corresponding infinite-volume continuous six-dimensional momentum integration numerically. Therefore we plot the perturbative expression only in the range where the perturbative finite-volume effects are small, $t / a \lesssim 10 ;$ an example of a result is shown in Fig. 2 ,

\footnotetext{
${ }^{1}$ In infinite volume, $\omega_{\mathrm{pl}}^{2}=g^{2} T C_{A}(3 \Sigma / 2 \pi-1) / 6 a$, where $\Sigma$ is the constant in Eq. (3.12) 20].
} 


\subsection{Value in the large-time limit}

As mentioned, it is not easy to evaluate numerically the HCL-resummed perturbative expression in Eq. (3.7), once the time coordinate becomes large: $\epsilon$ should be decreased, whereby the integrand becomes strongly peaked; and one should replace the finite-volume sums with infinite-volume momentum integrals, whereby the numerical cost increases. To get a handle on this limit we can, however, proceed in another way, without making use of the contour trick, and thereby obtain the correct version of Eq. (2.11) on an infinite spatial lattice. The starting point is then Eq. (2.10).

For infinitesimally small $\epsilon$, the small- $p^{0}$ behaviour of $\Pi_{E}$ reads (cf. Eq. (3.10) )

$$
\Pi_{E}\left(p^{0}+i 0^{+}, \tilde{\mathbf{p}}\right)=2 g^{2} C_{A} T\left[\frac{\Sigma}{4 \pi a}+i \pi p^{0} \int \mathrm{d} \mathbf{q} \frac{1}{\tilde{\mathbf{q}}^{2}} \delta\left(\frac{\tilde{\mathbf{p}} \cdot \stackrel{\circ}{\mathbf{q}}}{\sqrt{\tilde{\mathbf{q}}^{2}}}\right)\right]+\mathcal{O}\left(\left(p^{0}\right)^{2}\right) .
$$

Making use of the definition in Eq. (3.5), the asymptotic value from Eq. (2.10) can be written as

$$
\operatorname{Im}\left[\frac{V_{\mathrm{cl}}^{(2)}(\infty, r)}{g^{2} T}\right]=-\frac{\pi C_{F} C_{A}^{2}}{\beta} \int_{0}^{1} \mathrm{~d}^{3} \mathbf{x} \frac{1-\cos \left(\pi x_{3} r / a\right)}{\left(\tilde{\mathbf{x}}^{2}+C_{A}^{2} \Sigma / \pi \beta\right)^{2}} \int_{-1}^{1} \mathrm{~d}^{3} \mathbf{y} \frac{\delta(\tilde{\mathbf{x}} \cdot \mathbf{y})}{\left(\tilde{\mathbf{y}}^{2}\right)^{1 / 2}},
$$

where we have gone over to a notation where the integration variables are made dimensionless by going to lattice units, and the integration range is restricted to the unit box and its reflections:

$$
\tilde{x}_{i} \equiv 2 \sin \left(\frac{\pi x_{i}}{2}\right), \quad \stackrel{\circ}{x}_{i} \equiv \sin \left(\pi x_{i}\right), \quad x_{i} \in(-1,1) .
$$

Moreover, we have made use of the symmetry of the integrand, in order to restrict the integration to positive $x_{i}$.

Among the eight octants of the $\mathbf{y}$-integration, the $\delta$-function gets realised in six only, and by changes of integration variables we can combine all the contributions together:

$$
\begin{aligned}
\operatorname{Im}\left[\frac{V_{\mathrm{cl}}^{(2)}(\infty, r)}{g^{2} T}\right] & =-\frac{2 \pi C_{F} C_{A}^{2}}{\beta} \int_{0}^{1} \mathrm{~d}^{3} \mathbf{x} \frac{3-\cos \left(\pi x_{1} r / a\right)-\cos \left(\pi x_{2} r / a\right)-\cos \left(\pi x_{3} r / a\right)}{\left(\tilde{\mathbf{x}}^{2}+C_{A}^{2} \Sigma / \pi \beta\right)^{2}} \times \\
& \times \int_{0}^{1} \mathrm{~d}^{3} \mathbf{y} \frac{\delta\left(\tilde{x}_{1} \stackrel{\circ}{y}_{1}+\tilde{x}_{2} \stackrel{\circ}{y}_{2}-\tilde{x}_{3} \stackrel{\circ}{y}_{3}\right)}{\left(\tilde{\mathbf{y}}^{2}\right)^{1 / 2}}
\end{aligned}
$$

It is now straightforward to carry out the integration over, say, $x_{3}$, to remove the $\delta$-function, and also to make use of the symmetry of the remaining integrand in $x_{1} \leftrightarrow x_{2}$. The 5dimensional integral left over can be evaluated numerically without too much trouble. The result is shown in Fig. 3, for $N_{\mathrm{c}}=3$ and the case $r / a=\infty$, when the cosine-term does not contribute. Values of Eq. (3.23) at finite $r / a$ can be found in Table 1 below. Comparing Table 1 with Fig. 3, we observe that for, say, $\beta=16$, the distance $r / a=4$ gives a value which is already relatively close to the asymptotic one. To summarise, in resummed perturbation theory $\operatorname{Im}\left[V_{\mathrm{cl}}^{(2)}(\infty, r)\right]$ is definitely non-zero at all $r \neq 0$. 


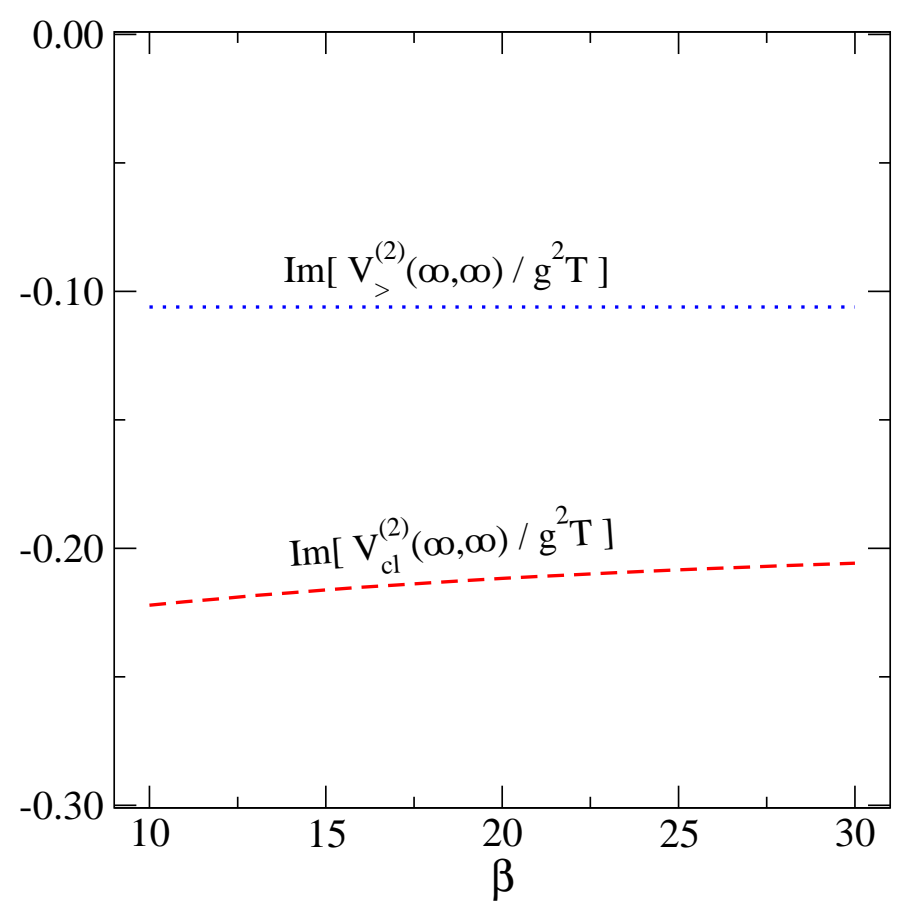

Figure 3: The asymptotic value of the real-time static potential $V_{\mathrm{cl}}(t, r)$, on an infinite lattice, to leading non-trivial order in HCL-resummed perturbation theory (Eq. (3.23)), for $N_{\mathrm{c}}=3$. For comparison, we also show the expression on the right-hand side of Eq. (2.11), which corresponds to the asymptotic value of the potential $V_{>}^{(2)}(t, r)$ in the full continuum quantum theory.

\section{Classical lattice gauge theory simulations}

The computation in the previous section was based on resummed perturbation theory, but it was only carried out to a fixed order. Let us try to estimate the expansion parameters of such a computation. Using continuum notation, the vertices of each new loop order bring in a factor $g^{2} T$. At the same time, the mass scales appearing in the dynamics are the ultraviolet cutoff scale, $\Lambda \sim 1 / a$, as well as the confinement scale of three-dimensional Yang-Mills theory, $m_{\mathrm{G}} \sim g^{2} T$ [5, 6]. The resummation accounts for the dominant influence of the hard cutoff scale $\Lambda$ on the dynamics of the infrared modes, and is associated with corrections of the type $g^{2} T / \Lambda \sim 1 / \beta$. However, it does not account for the self-interactions of the infrared modes, which may lead to an expansion parameter of the type $g^{2} T / m_{\mathrm{G}} \sim 1$. Therefore, we would like to compare the resummed perturbative result with a non-perturbative numerical computation.

The practical procedure of generating classical gauge field configurations is the following [17. Since our observable will be gauge-invariant, we may choose a gauge; it is convenient to work in the temporal gauge, $U_{t}(\mathbf{x}, t)=\mathbb{1}$. Here $U_{\mu}$ is an $\mathrm{SU}\left(N_{\mathrm{c}}\right)$ link matrix. The canonical degrees of freedom are the spatial link variables $U_{i}(\mathbf{x}, t)$ and the matrix-valued "canonical 
momenta" $E_{i}(\mathbf{x}, t)$, which transform in the adjoint representation. We denote the generators of the gauge group by $T^{a}$, and assume them normalised through $\operatorname{Tr}\left[T^{a} T^{b}\right]=\delta^{a b} / 2$. Furthermore, $E_{i} \equiv E_{i}^{a} T^{a}, S_{i j}(\mathbf{x}) \equiv U_{j}(\mathbf{x}) U_{i}(\mathbf{x}+j) U_{j}^{\dagger}(\mathbf{x}+i)$, where $i \equiv a \hat{e}_{i}$, and $U_{-i}(\mathbf{x}) \equiv U_{i}^{\dagger}(\mathbf{x}-i)$.

With this notation, the procedure starts by generating initial configurations (at time $t=0$ ) according to the partition function [17]

$$
\mathcal{Z}=\int \mathcal{D} U_{i} \mathcal{D} E_{i} \delta(G) \exp \left\{-\beta \sum_{\mathbf{x}}\left[\sum_{i<j}\left(1-\frac{1}{C_{A}} \operatorname{Re} \operatorname{Tr} P_{i j}\right)+\sum_{i} \operatorname{Tr}\left(E_{i}^{2}\right)\right]\right\},
$$

where $P_{i j}$ is the spatial plaquette, and the Gauss law function reads

$$
G(\mathbf{x}, t)=\sum_{i}\left[E_{i}(\mathbf{x}, t)-U_{-i}(\mathbf{x}, t) E_{i}(\mathbf{x}-i, t) U_{-i}^{\dagger}(\mathbf{x}, t)\right] .
$$

To obtain configurations extending to $t>0$, we solve the equations of motion

$$
\begin{aligned}
a \partial_{t} U_{i}(\mathbf{x}, t) & =i\left(2 C_{A}\right)^{\frac{1}{2}} E_{i}(\mathbf{x}, t) U_{i}(\mathbf{x}, t) \\
a \partial_{t} E_{i}^{b}(\mathbf{x}, t) & =-\left(\frac{2}{C_{A}}\right)^{\frac{1}{2}} \operatorname{Im} \operatorname{Tr}\left[T^{b} U_{i}(\mathbf{x}, t) \sum_{|j| \neq i} S_{i j}^{\dagger}(\mathbf{x}, t)\right]
\end{aligned}
$$

These four-dimensional configurations are then used for evaluating the real-time observables. In all that follows, we fix $C_{A}=N_{\mathrm{c}}=3$, even though we have also carried out some simulations at $N_{\mathrm{c}}=2$ as a crosscheck.

It is worth stressing that in Eqs. (4.3), (4.4), the lattice spacing is finite in spatial directions only. In practice, of course, the time direction needs to be discretised as well, but with a very small lattice spacing, $a_{t} \ll a$. As a check of the time evolution, it is useful to control the conservation of the Gauss law and of the total energy.

To specify the observable to measure, we adopt the definition in Eq. (2.5) as our nonperturbative starting point. The object appearing here is a specific analytic continuation of the Euclidean Wilson loop, and corresponds formally to a time ordering generally denoted with the subscript $(\ldots)_{>}[22]$ :

$$
C_{>}(t, r) \equiv C_{E}(i t, r) .
$$

At the same time, the classical $(\hbar \rightarrow 0)$ part of the analytic continuation, which we denote by $C_{\mathrm{cl}}(t, r)$, is independent of time ordering $\left(\lim _{\hbar \rightarrow 0} C_{>}=\lim _{\hbar \rightarrow 0} C_{<}=\lim _{\hbar \rightarrow 0}\left[C_{>}+C_{<}\right] / 2\right)$. In fact, $C_{\mathrm{cl}}(t, r)$ is nothing but the classical Wilson loop, defined in Minkowski time. (Note that having chosen the gauge $U_{t}=\mathbb{1}$, the classical Wilson loop amounts really to a two-point correlation function of two spatial Wilson lines, both of which are local in time.) The classical static potential is then measured from

$$
i \partial_{t} C_{\mathrm{cl}}(t, r) \equiv V_{\mathrm{cl}}(t, r) C_{\mathrm{cl}}(t, r) .
$$

It turns out that $C_{\mathrm{cl}}(t, r)$ is real for all times (within statistical errors), and slowly decaying. Therefore, $V_{\mathrm{cl}}(t, r)$ is purely imaginary, with a negative imaginary part. 


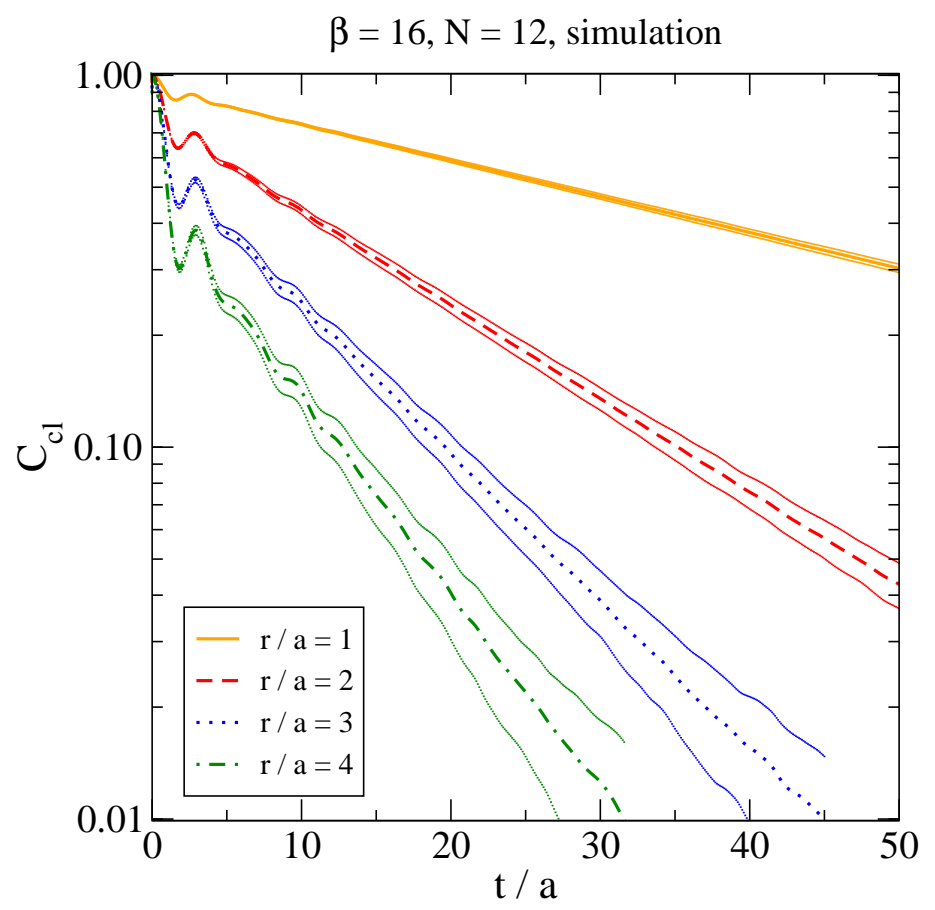

Figure 4: The classical Wilson loop, measured with classical lattice gauge theory simulations, as a function of time in units of the spatial lattice spacing, for $\beta=16, N=12, N_{\mathrm{c}}=3$.

The technical implementation of our simulation follows earlier work [17, 18]. However, to speed up thermalization, we have implemented the idea mentioned in ref. [23], whereby the link variables $U_{i}$ are first pre-thermalized with regular Monte Carlo techniques in the dimensionally reduced $\mathrm{SU}(3)+$ adjoint Higgs theory (we use the code described in ref. [24]). Since it is non-trivial to match the parameters of that theory and our effective theory exactly, those configurations are not yet fully thermalized. However, this is not a problem, they now need only to be evolved for a short time à la refs. [17, 18], in order to reach the correctly thermalized configurations corresponding to the exact parameters of Eq. (4.1).

We have carried out simulations mostly with $\beta=16$; since analytic HCL predictions also refer to a finite value of $\beta$, there is no need to carry out a continuum extrapolation (cf. Fig. 31). As typical lattice extents we have used $N=12$ and $N=16$; the difference of the results between these two is only at the percent level (cf. Table 1 below). The time variable is discretised with a spacing $a_{t}$, with a value $a_{t} / a=0.01$; measurements are recorded every 10th time step. We stress that thermalization is only carried out in the beginning, while the subsequent time evolution is deterministic and follows Eqs. (4.3), (4.4).

A representative result for the classical Wilson loop is shown in Fig. 4. The corresponding potential, extracted from Eq. (4.6), is shown in Fig. 5. The result can be compared with Fig. 2, showing the HCL-resummed perturbative prediction with the same parameter values. The general shapes are seen to match each other to a remarkable degree. On closer inspec- 


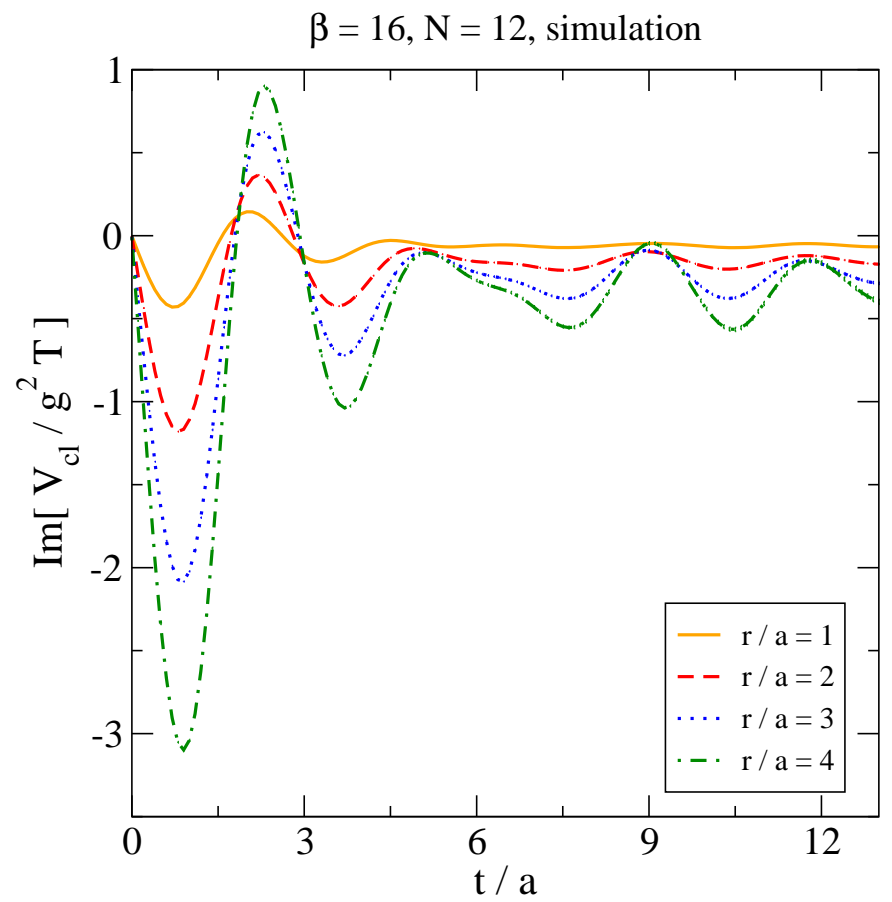

Figure 5: The imaginary part of the real-time static potential, measured with classical lattice gauge theory simulations, as a function of time in spatial lattice units (with the same parameter values as in Fig. (4). Vertical lines indicate statistical errors, but they are almost invisible in this time range.

tion, however, the amplitude of the oscillations is larger in the simulation; the frequency of oscillations is smaller (the oscillation period is larger); and the absolute value of the potential is larger (the imaginary part is more negative).

In order to quantify the difference, we note that at large times, the potential obtains a constant value (or, in terms of the Wilson loop, $C_{\mathrm{cl}}$ decays exponentially, cf. Fig. 44). We estimate this value by fitting a constant to data in the range $t / a=15 \ldots 30$, where initial transients have died out, yet the statistical errors are still relatively small for all parameter values that we have used. The results of the fits are shown in Table 1. Unfortunately, statistical errors rapidly increase with $r / a$, and we are not able to go to large enough values for the $r$-dependence to have flattened off. Nevertheless, the values at $r / a=4$ already indicate that the asymptotic value is larger (in absolute magnitude) than the analytic HCL estimate, by some $50-100 \%$. The fact that there thus appears to be somewhat more "damping" in the non-perturbative classical dynamics than in the HCL estimate is not surprising: other observables have yielded indications of a similar pattern [20].

We have also carried out so-called HTL simulations, both with the implementation based on treating the velocities of the hard particle degrees of freedom with spherical harmonics [19, 25], and through a discretization based on platonic solids [26]. The HTL-simulations introduce a new parameter, which we refer to as $m_{\mathrm{D}}^{\text {(bare) }}$. For small $m_{\mathrm{D}}^{\text {(bare) }}$, say $a m_{\mathrm{D}}^{\text {(bare) }} \lesssim 0.2$, the 


\begin{tabular}{|c|c|c|c|c|l|l|l|l|}
\hline \multicolumn{5}{|c|}{ parameters } & \multicolumn{4}{c|}{ value of $\operatorname{Im}\left[V_{\mathrm{cl}}(\infty, r) / g^{2} T\right]$} \\
\hline$\beta$ & $N$ & $a m_{\mathrm{D}}^{\text {(bare) }}$ & method & confs & $r / a=1$ & $r / a=2$ & $r / a=3$ & $r / a=4$ \\
\hline \hline 16.0 & 12 & 0.00 & simulation & 200 & $-0.060(2)$ & $-0.156(8)$ & $-0.246(26)$ & $-0.319(56)$ \\
\hline 16.0 & 16 & 0.00 & simulation & 160 & $-0.059(2)$ & $-0.155(8)$ & $-0.245(22)$ & $-0.326(48)$ \\
\hline 16.0 & 12 & 0.21 & simulation & 200 & $-0.059(2)$ & $-0.147(7)$ & $-0.229(23)$ & $-0.297(51)$ \\
\hline 16.0 & 12 & 0.35 & simulation & 182 & $-0.030(2)$ & $-0.064(5)$ & $-0.096(12)$ & $-0.118(21)$ \\
\hline 13.5 & 12 & 0.25 & simulation & 142 & $-0.071(2)$ & $-0.174(10)$ & $-0.270(33)$ & $-0.341(97)$ \\
\hline \hline 16.0 & $\infty$ & 0.00 & analytic & - & -0.0601 & -0.1145 & -0.1507 & -0.1737 \\
\hline
\end{tabular}

Table 1: The asymptotic values $\operatorname{Im}\left[V_{\mathrm{cl}}(\infty, r) / g^{2} T\right]$, obtained by fitting a constant to data in the range $t / a=15 \ldots 30$. The numbers in parentheses indicate the uncertainties of the last digits. The cases $a m_{\mathrm{D}}^{\text {(bare) }}=0.00$ refer to the classical theory without HTL degrees of freedom. The bottom row gives the perturbative values from Eq. (3.23); the perturbative result at $r / a=\infty$ is -0.2152 .

results are practically identical with those of the classical simulations (cf. Table 1). With increasing $m_{\mathrm{D}}^{\text {(bare) }}$, say $a m_{\mathrm{D}}^{\text {(bare) }} \gtrsim 0.35$, we see some discrepancies; in particular, the asymptotic value $\operatorname{Im}\left[V_{\mathrm{cl}}(\infty, r)\right]$ decreases in absolute magnitude (cf. Table 1), as one would expect in a situation where a lattice-induced dynamical Debye screening is overtaken by a continuumlike parameter (cf. Fig. 3). At the same time, this method is not really bringing us closer to the physical continuum limit (i.e. the continuum limit of the quantum theory), since in that situation the bare parameter $\left[m_{\mathrm{D}}^{(\text {bare })}\right]^{2}$ should in fact become negative as $\beta$ is increased, in order to cancel ultraviolet divergences from the dynamics. Unfortunately, the implementation of HTL-simulations that we have followed, based on refs. [19, 26], does not allow to simulate at $\left[m_{\mathrm{D}}^{\text {(bare) }}\right]^{2}<0$, and a single bare parameter would in any case not allow to renormalise all the observables that can be measured with classical lattice gauge theory [15, 16]. Therefore, we omit a more detailed discussion of the HTL-simulations from here.

\section{Conclusions}

The purpose of this paper has been to elaborate on the fact that the finite-temperature realtime static potential extracted from an analytic continuation of the Euclidean Wilson loop, which can (at least to some order in perturbation theory) be inserted into a Schrödinger-type equation governing the behaviour of a certain heavy quarkonium Green's function, contains an imaginary part. As discussed elsewhere [14], this imaginary part has an impact on the heavy quarkonium spectral function at temperatures above a few hundred $\mathrm{MeV}$, introducing a width to (the tip of) the resonance peak.

Physically, the imaginary part implies that quarkonium at high temperatures should not be thought of as a stationary state. Rather, the norm of its wave function decays exponentially 
with time.

The imaginary part emerges from Bose-enhanced infrared dynamics and, in field-theory language, is classical in nature (in particle language, it corresponds to a net disappearance of low-energy off-shell gauge particles, due to inelastic $2 \rightarrow 1$ and $1 \rightarrow 2$ scatterings with the hard particles in the plasma). We have computed the imaginary part with Hard Classical Loop resummed perturbation theory, and with non-perturbative classical lattice gauge theory simulations. The comparison of the results, Fig. 2 and Fig. 5, or Table 1, shows reasonable qualitative agreement. We conclude that non-perturbative colour-magnetic fields do not play a dominant role for the imaginary part of the real-time static potential; however, the nonperturbative corrections, together with higher-order perturbative terms, are important on the quantitative level, bringing about some $50-100 \%$ increase in the absolute value of the imaginary part at large times (for $\beta=16$ ), akin in magnitude to the correction observed for the static Debye screening mass [24]. In any case, our study confirms that an imaginary part exists, and suggests that Hard Loop perturbation theory presumably provides for a reasonable first estimate for it also in the full quantum theory.

For physical applications, such as determining the quarkonium spectral function, it is essential to use the full quantum theory, rather than the classical one. Moreover, it is convenient to use dimensional regularization. Finally, as argued in ref. [14, the static potential should be evaluated at $t \gg r$. The perturbative static potential in this limit, $V_{>}^{(2)}(\infty, r)$, including both a real and an imaginary part, can be found in Eqs. (4.3), (4.4) of ref. [12], and has already been employed for estimating the quarkonium spectral function in ref. [14]. Increasing the imaginary part by some $50-100 \%$ in the results of ref. [14] lowers and widens the quarkonium peak, but the effect is not dramatic; in general, it appears that the spectral function is more sensitive to the real than the imaginary part of the real-time static potential.

As the next step of our program, we would therefore like to get a non-perturbative handle also on the real part of the real-time static potential, $V_{>}(t, r)$, entering the Schrödingerequation. In particular, it would be important to clarify its connection to the other static potentials that are being used for studying the spectral function of heavy quarkonium in high-temperature QCD (for recent work and references see, e.g., ref. [27]).

\section{Acknowledgements}

We wish to thank P. Romatschke for fruitful collaboration during initial stages of this investigation. M.L. acknowledges useful discussions with Seyong Kim. Our work was partially supported by the BMBF project Hot Nuclear Matter from Heavy Ion Collisions and its Understanding from $Q C D$. 


\section{References}

[1] W.E. Caswell and G.P. Lepage, Phys. Lett. B 167 (1986) 437.

[2] A. Pineda and J. Soto, Nucl. Phys. B (Proc. Suppl.) 64 (1998) 428 hep-ph/9707481; N. Brambilla, A. Pineda, J. Soto and A. Vairo, Nucl. Phys. B 566 (2000) 275 hep-ph/9907240.

[3] M. Beneke, hep-ph/9911490.

[4] N. Brambilla, A. Pineda, J. Soto and A. Vairo, Rev. Mod. Phys. 77 (2005) 1423 hep-ph/0410047.

[5] A.D. Linde, Phys. Lett. B 96 (1980) 289.

[6] D.J. Gross, R.D. Pisarski and L.G. Yaffe, Rev. Mod. Phys. 53 (1981) 43.

[7] P. Arnold, D. Son and L.G. Yaffe, Phys. Rev. D 55 (1997) 6264 [hep-ph/9609481].

[8] D. Bödeker, Phys. Lett. B 426 (1998) 351 hep-ph/9801430.

[9] S. Nadkarni, Phys. Rev. D 33 (1986) 3738; A.K. Rebhan, Nucl. Phys. B 430 (1994) 319 hep-ph/9408262; E. Braaten and A. Nieto, Phys. Rev. Lett. 74 (1995) 3530 hep-ph/9410218; P. Arnold and L.G. Yaffe, Phys. Rev. D 52 (1995) 7208 hep-ph/9508280.

[10] O. Jahn and O. Philipsen, Phys. Rev. D 70 (2004) 074504 hep-lat/0407042.

[11] G. Cuniberti, E. De Micheli and G.A. Viano, Commun. Math. Phys. 216 (2001) 59.

[12] M. Laine, O. Philipsen, P. Romatschke and M. Tassler, JHEP 03 (2007) 054 hep-ph/0611300.

[13] R.D. Pisarski, Phys. Rev. Lett. 63 (1989) 1129; J. Frenkel and J.C. Taylor, Nucl. Phys. B 334 (1990) 199; E. Braaten and R.D. Pisarski, Nucl. Phys. B 337 (1990) 569; J.C. Taylor and S.M.H. Wong, Nucl. Phys. B 346 (1990) 115.

[14] M. Laine, JHEP 05 (2007) 028 arXiv:0704.1720].

[15] D. Bödeker, L.D. McLerran and A. Smilga, Phys. Rev. D 52 (1995) 4675 hep-th/9504123.

[16] P. Arnold, Phys. Rev. D 55 (1997) 7781 hep-ph/9701393.

[17] D.Y. Grigoriev and V.A. Rubakov, Nucl. Phys. B 299 (1988) 67; J. Ambjørn, T. Askgaard, H. Porter and M.E. Shaposhnikov, Nucl. Phys. B 353 (1991) 346; J. Ambjørn and A. Krasnitz, Nucl. Phys. B 506 (1997) 387 hep-ph/9705380]. 
[18] G.D. Moore and K. Rummukainen, Phys. Rev. D 61 (2000) 105008 hep-ph/9906259.

[19] D. Bödeker, G.D. Moore and K. Rummukainen, Phys. Rev. D 61 (2000) 056003 hep-ph/9907545.

[20] D. Bödeker and M. Laine, Phys. Lett. B 416 (1998) 169 hep-ph/9707489.

[21] K. Farakos, K. Kajantie, K. Rummukainen and M. Shaposhnikov, Nucl. Phys. B 442 (1995) 317 hep-lat/9412091.

[22] M. Le Bellac, Thermal Field Theory (Cambridge University Press, Cambridge, 2000).

[23] G.D. Moore, Nucl. Phys. B 480 (1996) 657 hep-ph/9603384].

[24] A. Hart, M. Laine and O. Philipsen, Nucl. Phys. B 586 (2000) 443 [hep-ph/0004060].

[25] M. Hindmarsh and A. Rajantie, Phys. Rev. D 64 (2001) 065016 [hep-ph/0103311].

[26] A. Rebhan, P. Romatschke and M. Strickland, Phys. Rev. Lett. 94 (2005) 102303 hep-ph/0412016; JHEP 09 (2005) 041 hep-ph/0505261.

[27] Y. Maezawa, N. Ukita, S. Aoki, S. Ejiri, T. Hatsuda, N. Ishii and K. Kanaya [WHOT-QCD Collaboration], Phys. Rev. D 75 (2007) 074501 hep-lat/0702004; M. Döring, K. Hübner, O. Kaczmarek and F. Karsch, Phys. Rev. D 75 (2007) 054504 hep-lat/0702009]; A. Mócsy and P. Petreczky, arXiv:0705.2559 [hep-ph]; W.M. Alberico, A. Beraudo, A. De Pace and A. Molinari, arXiv:0706.2846 [hep-ph]. 